Please cite this paper as follows:

R. Teixeira Pinto; M. Aragues-Penalba; O. Gomis-Bellmunt; A. Sumper, "Optimal Operation of DC Networks to Support Power System Outage Management," in IEEE Transactions on Smart Grid, vol.PP, no.99, pp.1-1 doi: 10.1109/TSG.2016.2586024 


\title{
Optimal Operation of DC Networks to Support Power System Outage Management
}

\author{
R. Teixeira Pinto, Member, IEEE, M. Aragüés-Peñalba, Student Member, IEEE, O. Gomis-Bellmunt, Senior \\ Member, IEEE, and A. Sumper, Member, IEEE
}

\begin{abstract}
The penetration of dc networks for different applications in power systems is increasing. This paper presents a novel methodology for security-constrained optimal power flow (SCOPF) operation of a power system, such as a smart grid or a supergrid, with an embedded dc network. The methodology demonstrates that de networks can be operated to provide support to ac systems, increasing its security of supply and resilience in case of outages, while reducing operational costs. Moreover, the outage management support can be achieved via a preventive SCOPF - i.e. the combined network stays N-1 secure after outages without need for further control action - or via a corrective SCOPF, by using the fast controls of the ac-dc converters to react to the contingencies. The methodology relies on the construction of a binary outage matrix and optimizes only the control variables of the ac and dc networks. It was successfully tested in system with 12 buses and in the IEEE30 network with 35 buses. Operational savings of up to $1 \%$ and $0.52 \%$ were obtained for the first and second networks, respectively, while network violations for the $\mathrm{N}-1$ contingency scenarios were completely eliminated in the first and reduced by $70 \%$ in the former.
\end{abstract}

Index Terms-MTDC networks, supergrids, SCOPF, outage management, restoration algorithms.

\section{INTRODUCTION}

I $\mathrm{N}$ the next two decades circa $80 \%$ of all the generated electricity is expected to be converted from ac to dc for final utilization mainly due to the increasing presence of electric motor drives and power electronic converters (nowadays only $30 \%$ is converted) [1]. Nowadays, direct current networks are present in microgrids and smart grids, in electronic power distribution systems and in supergrids dedicated to integrating continental renewable resources [2-4]. Therefore, several studies have proposed tools to achieve optimal power flow (OPF) in combined ac-dc networks [5-8].

When a dc network is integrated into existing ac networks, the control flexibility of the ac-dc converters can alter usual power flow regimes. The power flow algorithms for combined ac-dc systems can be simultaneous or unified, i.e. the execution of the ac and dc networks power flow is done in parallel or, alternatively, they can be sequential, i.e. the power flows are solved iteratively until convergence is reached. The simultaneous approach is used in [5], whereas the iterative approach is used in [9]. Hitherto, the usual approach to the ac-dc OPF problem is to add all the dc power flow equations into a regular ac OPF problem as a set of linear and non-linear equality constraints, which is acceptable for small dc networks.

The response to emergency situations in power systems is usually divided into two main categories, the reliability planning level and the contingency planning level, which differ based on the time horizon concerned. The first category, the reliability planning, has a longer planning horizon of usually circa 5 to 10 years and regards the decisions needed to guarantee a reliable system operation and expansion taking fault cases into account $[10,11]$. On the other hand, the realtime response to emergencies, dealt with in this paper, belongs to the contingency planning level.

Nowadays, the fact that transmission systems are being expanded using both ac and de technologies introduces complexity and challenges in the power system operation and control, but can also contribute to the security of the system, enhancing power supply security [12], mitigating the ac transmission congestion [13] and helping in the restoration process in case of outages [14]. In case of contingencies in the power system, all the system operator ( $\mathrm{SO}$ ) attention is given to restore the power supply as fast as possible. The actions undertaken to deal with real-time emergency situations are known as power system restoration (PSR) problem [15]. The actions when a power system is faced which a contingency are normally divided into states, shown in Figure 1 together with and which approach can be used for each state.

The main power system states are: Normal, Pre-outage and Outage [10, 16], In extremis [17] and Restorative [15, 18].

Some studies exist for analysing one or some of the before described states in hybrid ac-dc systems (for instance, [19], which takes into account corrective actions, or [20], which considers preventive actions). The present work deals with the security constrained operation of a combined ac-dc power system during the states 1 to 4 . One of the challenges identified in SCOPF analysis is reducing the size of the optimization problem derived $[21,22]$. In general, depending on how constrained the OPF problem search space is, it is better to have less constraints as well as less variables to optimize [23]. However, the usual approach increases not only the number of constraints, but also the number of variables the OPF problem must optimize, and it may not be acceptable in case larger

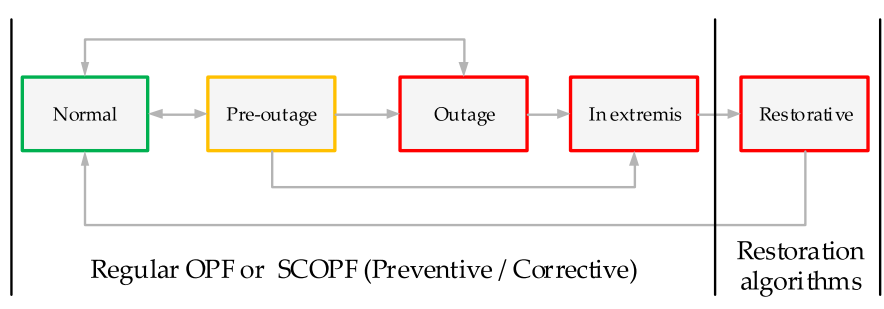

Fig. 1. The five possible power system states according to [17]. 
dc networks are embedded in the ac networks. Therefore, this paper presents a novel methodology which reduces the number of constraints as well as the number of variables the combined ac-dc OPF problem must consider during emergency situations.

The paper is organized as follows: Section II explains the proposed methodology. Section IV presents the results for the normal OPF, and preventive and corrective securityconstrained optimal power flow (SCOPF) operation of a 9-bus ac system with a 3-node dc network (here addressed as the $9 A C+3 D C$ network), and for the IEEE 30-bus ac system with a 5-node dc network (here addressed as 30AC+5DC network). Finally, conclusions are given and the future work is presented.

\section{Methodology}

In the proposed methodology, depicted in Fig. 2, only control variables are optimized. The methodology works as follows: for a given initial control vector, the dc state variables are solved first via a dc power-flow routine.

Afterwards, the ac state variables are solved via an ac power-flow routine. As they converge separately, there is no need for iterations between the two power-flow routines since the outputs of the dc power flow (described in [24-26]) are used as inputs in the ac power flow. Both the ac and dc power flows are solved using Newton-Raphson. The only iteration in the proposed methodology lies in the SCOPF algorithm which relies on an optimization routine.

The state-variables in dc networks are the direct voltages of the power electronic converters in power regulation mode, which means their direct voltages are not known beforehand. On the other hand, the control variables $\mathbf{u}_{d c}$ in dc networks are the active power of the converters in power regulation mode and the direct voltage of the converters in voltage regulation mode, i.e. the converters controlling the dc network voltage.

In the ac network, the state variables are the voltage amplitudes and load angles at the grid PQ nodes, together with the load angles at the PV nodes. Finally, in the ac network side, the control variables $\mathbf{u}_{a c}$ are the voltages and reactive power of ac generators or ac-dc converters, and the active power of ac generators. The control variables vector then becomes:

$$
\begin{aligned}
& \mathbf{u}_{1 \times\left(2 g_{n}+2 c_{n}-1\right)}=\left[\begin{array}{ll}
\mathbf{u}_{\mathbf{a c}} & \mathbf{u}_{\mathbf{d c}}
\end{array}\right]= \\
& {\left[\begin{array}{lllllll}
\mathbf{P}_{g, 1} & \mathbf{Q}_{g, 1} & \mathbf{V}_{g, 1} & \mathbf{P}_{c, 1} & \mathbf{Q}_{c, 1} & \mathbf{V}_{c, 1} & \mathbf{U}_{c, 1}
\end{array}\right]}
\end{aligned}
$$

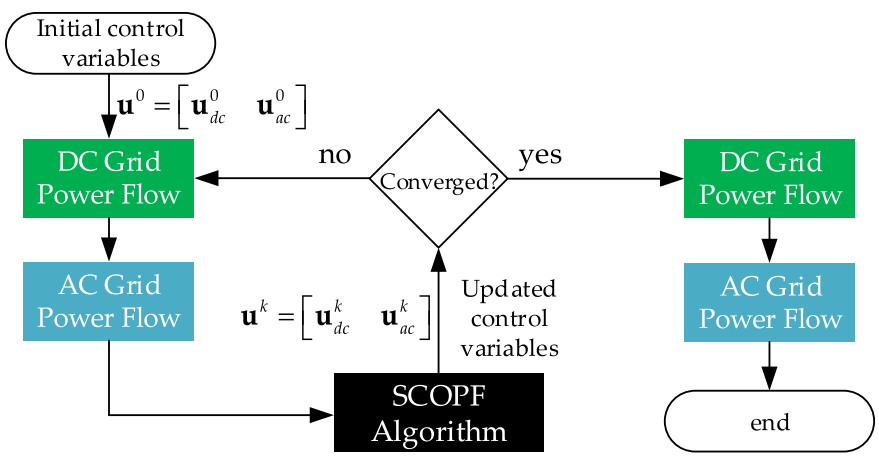

Fig. 2. Proposed OPF methodology for combined ac-dc networks. where, $g_{n}$ is the number of ac generators in the network and $c_{n}$ is the numbers of ac-dc converters in the networks. The dimension of the control vector $\mathbf{u}$ is equal:

$$
[\mathbf{u}]=2 g_{n}+2 c_{n}-1
$$

since the active power of the slack ac generator cannot be optimized.

The objective function, $f$, for the SCOPF algorithm is taken as the unit commitment, i.e. minimize the total generation costs with a polynomial second order model given as:

$$
f=\min \left(\sum_{i=1}^{n} a_{g_{i}} \cdot P_{g_{i}, 1}^{2}+b_{g_{i}} \cdot P_{g_{i}, 1}+c_{g_{i}}\right)
$$

where, $g_{n}$ is the number of ac generators in the network.

Other objective functions, e.g. maximize the total social welfare in a pool market or minimize the overall system losses, or even a multi-objective optimization are also possible.

When a solution is found, the two power-flow routines are performed one last time to obtain the state variables for the final control variables.

The SCOPF algorithm is the core of the optimization process. It updates the control variables until convergence is reached and a solution is found. During the optimization step, the SCOPF algorithm tries to make sure the resulting solution of the combined ac-dc network is $\mathrm{N}-1$ secure.

\section{A. SCOPF algorithm}

The proposed methodology to operate combined ac-dc networks can be performed in three different modes: firstly, as a regular OPF where $\mathrm{N}-1$ security is not considered; secondly, as a preventive SCOPF where all the necessary measurements to possible component outages are taken preventively to make the systems N-1 secure and; thirdly, as a corrective SCOPF where the power electronic converters change their operational setpoint following an outage so that the network is N-1 secure.

In this work, four main component outages were considered: ac lines, dc lines, ac generators and ac-dc converters. For each system state during an outage of one system component - $\mathrm{N}$ states in total - the functioning status of a system component is represented through a binary variable, $\mathbf{x} \in\{\mathbf{0}, \mathbf{1}\}$, where the component status is 1 if it is in operation and 0 otherwise. Therefore, $\mathbf{x}$ is a binary outage matrix written as:

$$
\mathbf{x}_{E \times(N+1)}=\left(\begin{array}{ccc}
x_{a c_{1}, 1} & \cdots & x_{a c_{1}, N+1} \\
\ldots & \cdots & \ldots \\
x_{a c_{n}, 1} & \cdots & x_{a c_{n}, N+1} \\
x_{d c_{1}, 1} & \cdots & x_{d c_{1}, N+1} \\
\cdots & \cdots & \cdots \\
x_{d c_{n}, 1} & \cdots & x_{d c_{n}, N+1} \\
x_{g_{1}, 1} & \cdots & x_{g_{1}, N+1} \\
\cdots & \cdots & \cdots \\
x_{g_{n}, 1} & \cdots & x_{g_{n}, N+1} \\
x_{c_{1}, 1} & \cdots & x_{c_{1}, N+1} \\
\cdots & \cdots & \cdots \\
x_{c_{n}, 1} & \cdots & x_{c_{n}, N+1}
\end{array}\right)
$$


where, $x_{a c_{i}, j}$ is the status of ac line $\mathrm{i}$ during system outage scenario $\mathrm{j}$ and $a c_{n}$ is the total number of ac lines in the network; $x_{d c_{i}, j}$ is the status of dc line $\mathrm{i}$ during system outage scenario $\mathrm{j}$ and $d c_{n}$ is the total number of $\mathrm{dc}$ lines in the network; $x_{g_{i}, j}$ is the status of generator i during system outage scenario $\mathrm{j}$ and $g_{n}$ is the total number of generators in the network and; $x_{c_{i}, j}$ is the status of converter $\mathrm{i}$ during system outage scenario $\mathrm{j}$ and $c_{n}$ is the total number of ac-dc converters in the network.

The $\mathbf{x}$ matrix, which is composed of binary values, determines which component of the network is out of order during the contingency scenarios. The value of a component (connected or disconnected) is then used during the solution of the the power-flow equations. This is the reason why the resulting dimension of $\mathbf{x}$ is $E \times(N+1)$, where $E$ is the number of network elements included in the N-1 contingency analysis, as well as the number of possible single contingency scenarios. In the case where all system components are included in the $\mathrm{N}-1$ security analysis, then $E=N$. The last column in (4) accounts for the situation in which all system components are operating normally, without outages, as this is the scenario used for optimizing the system operation cost function.

\section{B. Normal OPF}

In case no security constraints are enforced and just a regular OPF is performed, then the number of outages to consider is zero and, therefore, the $\mathbf{x}$ matrix shown in (4) becomes $\mathbf{x}_{E \times 1}=\mathbf{1}$ (a vector of ones). In this scenario the control variables shown in (1) are optimized so that the total generation cost given in (3) are minimum.

\section{Preventive SCOPF}

When a preventive SCOPF is performed the set of control variables $\mathbf{u}$ are the same as for when a normal OPF is done. However, this set of control variables is employed in $\mathrm{N}+1$ power-flow calculations to make sure that when a system component has an outage, the network limits are still enforced.

In that case, since there will be $\mathrm{N}+1$ power-flow results, the object function is taken as:

$$
\left\{\begin{array}{l}
f=\min \left(\sum_{i=1}^{n} a_{g_{i}} \cdot P_{g_{i}, N+1}^{2}+b_{g_{i}} \cdot P_{g_{i}, N+1}+c_{g_{i}}\right) \\
\text { s.t. } \sum_{j=1}^{N} \mu_{j}=0
\end{array}\right.
$$

where $P_{g_{i}, N+1}$ is the active power of the ac generator $\mathrm{i}$ when all the network components are functional and; $\mu_{j}$ is the total number of system constraint violations when outage $\mathrm{j}$ takes place.

\section{Corrective SCOPF}

For the corrective SCOPF formulation, the same $\mathbf{x}-$ shown in (4) - is used as for the preventive SCOPF formulation. However, this time the number of optimization variables will change since the power electronic converters will have different set points for each of the possible system outages. Therefore, (1) is not anymore a vector but a matrix given as:

$$
\mathbf{u}_{(N+1) \times\left(g_{n}+c_{n}-1\right)}=\left(\begin{array}{ccc}
\mathbf{P}_{g, 1} & \cdots & \mathbf{P}_{g, N+1} \\
\mathbf{Q}_{g, 1} & \cdots & \mathbf{Q}_{g, N+1} \\
\mathbf{V}_{g, 1} & \cdots & \mathbf{V}_{g, N+1} \\
\mathbf{P}_{c, 1} & \cdots & \mathbf{P}_{c, N+1} \\
\mathbf{Q}_{c, 1} & \cdots & \mathbf{Q}_{c, N+1} \\
\mathbf{V}_{c, 1} & \cdots & \mathbf{V}_{c, N+1} \\
\mathbf{U}_{c, 1} & \cdots & \mathbf{U}_{c, N+1}
\end{array}\right)^{T}
$$

However, since in the corrective SCOPF only the power electronic converters set points can change, it follows that:

$$
\left\{\begin{array}{l}
\mathbf{P}_{g, 1}=\cdots=\mathbf{P}_{g, N+1} \\
\mathbf{Q}_{g, 1}=\cdots=\mathbf{Q}_{g, N+1} \\
\mathbf{V}_{g, 1}=\cdots=\mathbf{V}_{g, N+1}
\end{array}\right.
$$

which means the ac generators set points must remain the same for all the possible $\mathrm{N}+1$ scenarios, i.e. the $\mathrm{N}$ outages possibilities plus the normal operation condition.

When performing corrective SCOPF, it is commonly assumed that as power electronic converters react faster, the reaction of the ac generators can be neglected [13, 19, 21]. However, to include generators variables, e.g. Automatic Voltage Regulator (AVR) controllers, all that is need is to include these variables in the set of optimization variables for the corrective SCOPF algorihtm.

As it was the case with the preventive SCOPF formulation, there will be $\mathrm{N}+1$ power-flow results and the object function is the same as the one shown in (5).

\section{CAse Studies}

The proposed ac-dc SCOPF algorithm was tested in two distinct power systems: firstly, on an ac network with 9 nodes (based on MATPOWER data [27]) combined with a dc network with 3 nodes and, later, on the IEEE 30 bus network combined with a 5 node dc network. The layout of the first ac-dc system is shown in Figure 3, whereas the layout of the second ac-dc system is shown in Figure 4.

For both the ac networks, the data and operational limits are taken as given in MATPOWER [27]. For both dc networks, the direct voltage was chosen equal $\pm 200 \mathrm{kV}$, all ac-dc converters are rated equally at $100 \mathrm{MVA}$, the dc cables resistance is taken as $0.151 \Omega / \mathrm{km}$ (the cables lengths are given in Figure 3 and Figure 4) and the direct voltage normal operational range is assumed from 0.95 to $1.05 \mathrm{pu}$.

For the power flow in the ac and dc grids the transmission lines are modelled as a single pi-section and loads are considered of constant power. The ac-dc converters are considered lossless, but the losses on the dc grid are taken into consideration.

In both case studies, the following outages are considered:

1) contingencies on ac lines $\left(x_{a c_{i}, j}\right)$;

2) contingencies on dc lines $\left(x_{d c_{i}, j}\right)$;

3) contingencies ac generators $\left(x_{g_{i}, j}\right)$ and;

4) contingencies on ac-dc converters $\left(x_{c_{i}, j}\right)$. 


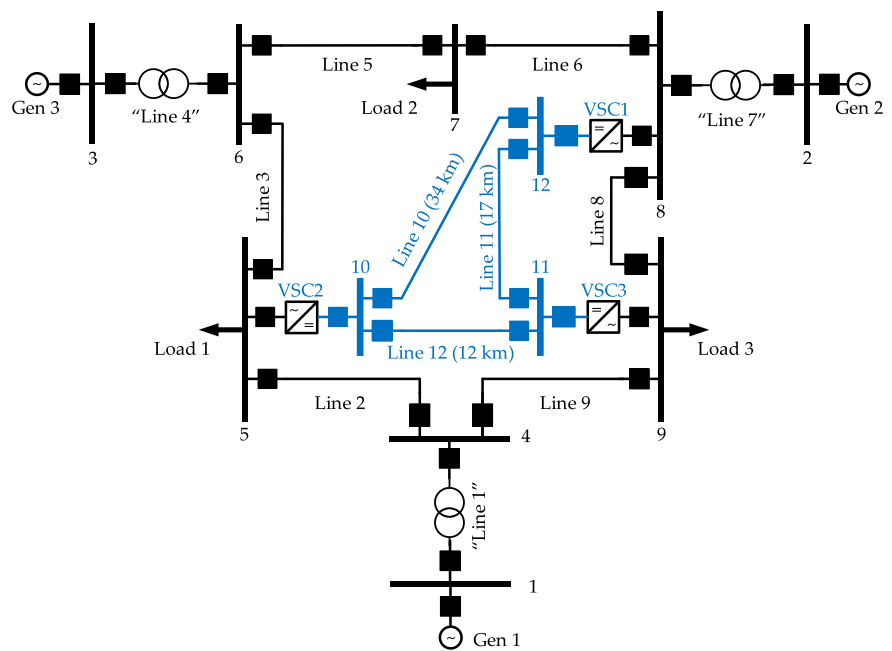

Fig. 3. Combined ac-de network with 9 ac and $3 \mathrm{dc}$ buses $(9 \mathrm{AC}+3 \mathrm{DC}$ network).

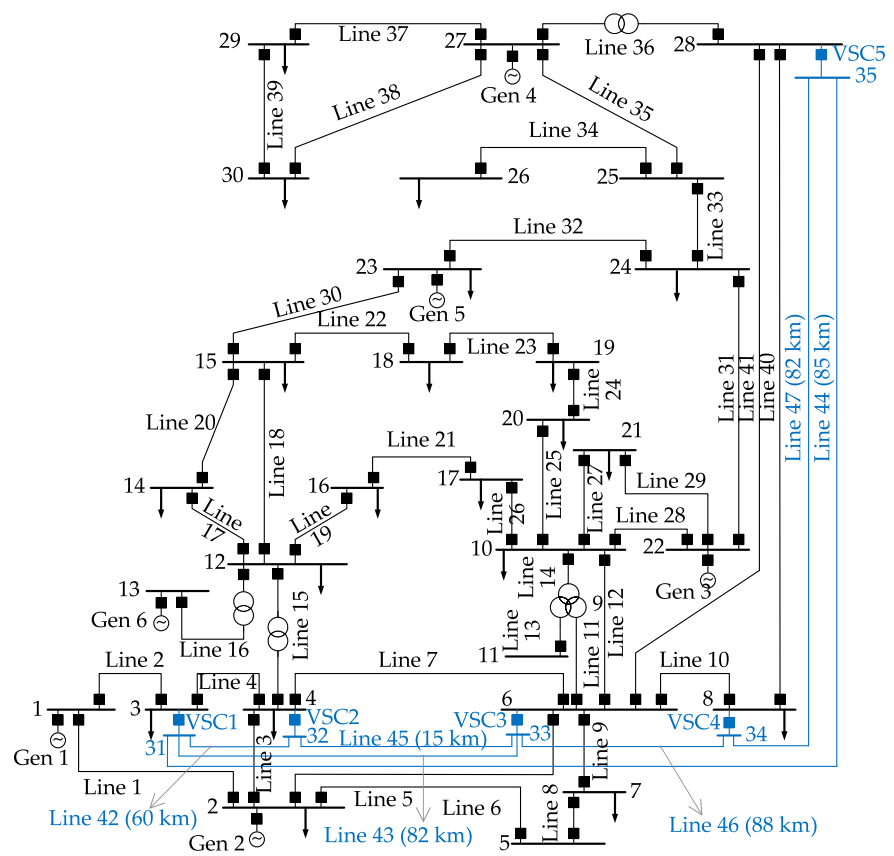

Fig. 4. Combined ac-dc network with 30 ac and $5 \mathrm{dc}$ buses $(30 \mathrm{AC}+5 \mathrm{DC}$ network).

Moreover, the SCOPF algorithm is constrained by the following network operational limits (inequality constraints):

1) maximum loadings on ac and dc lines;

2) minimum and maximum voltages on ac and dc buses and;

3) minimum and maximum power ratings of all ac generators and ac-dc converters.

As mentioned in (5), wherever a network operational limit is violated for a specified contingency scenario $\mathrm{j}$, then the value of $\mu_{j}$ is altered to reflect the total number of violations in that network condition.

For the smaller system, there are 18 possible N-1 contingency scenarios -9 ac lines, 3 dc lines, 3 ac generators and 3 ac-dc converters - whereas for the larger system there are 58 possible $\mathrm{N}-1$ contingency scenarios -41 ac lines, $6 \mathrm{dc}$ lines,
6 ac generators and 5 ac-dc converters.

In both systems the ac generators and the ac-dc converters are controlling their active power and their nodal voltage. The exceptions are both generators 1 , which are taken as the slack nodes in their respective ac networks and, therefore, can only control their nodal voltage; and both ac-dc converters 1, which are taken as the slack nodes for their respective dc network and, hence, control their direct voltage.

Therefore, according to (2) there will be 11 control variables in the smaller network and 21 in the larger network when the normal OPF or the preventive SCOPF are performed. Although the larger network has thrice the number of nodes than the smaller network, the optimization variables are barely doubled, as only control variables are optimized. Instead, if a corrective SCOPF is performed, the number of variables become 209 for the smaller network (due to the 18 possible $\mathrm{N}-1$ contingency scenarios), and 1239 for the larger network (due to the possible $58 \mathrm{~N}-1$ contingency scenarios).

Based on the aforementioned data for the smaller network, in the case of a preventive SCOPF, the $\mathbf{x}$ is a matrix can be written as:

$$
\mathbf{x}_{18 \times 19}=\left(\begin{array}{cccc}
x_{a c_{1}, 1}=0 & \cdots & x_{a c_{1}, 18}=1 & x_{a c_{1}, 19}=1 \\
\cdots & \cdots & \cdots & \cdots \\
x_{a c_{9}, 1}=1 & \cdots & x_{a c_{9}, 18}=1 & x_{a c_{9}, 19}=1 \\
x_{d c_{1}, 1}=1 & \cdots & x_{d c_{1}, 18}=1 & x_{d c_{1}, 19}=1 \\
x_{d c_{2}, 1}=1 & \cdots & x_{d c_{2}, 18}=1 & x_{d c_{2}, 19}=1 \\
x_{d c_{3}, 1}=1 & \cdots & x_{d_{3}, 18}=1 & x_{d c_{3}, 19}=1 \\
x_{g_{1}, 1}=1 & \cdots & x_{g_{1}, 18}=1 & x_{g_{1}, 19}=1 \\
x_{g_{2}, 1}=1 & \cdots & x_{g_{2}, 18}=1 & x_{g_{2}, 19}=1 \\
x_{g_{3}, 1}=1 & \cdots & x_{g_{3}, 18}=1 & x_{g_{3}, 19}=1 \\
x_{c_{1}, 1}=1 & \cdots & x_{c_{1}, 18}=1 & x_{c_{1}, 19}=1 \\
x_{c_{2}, 1}=1 & \cdots & x_{c_{2}, 18}=1 & x_{c_{2}, 19}=1 \\
x_{c_{3}, 1}=1 & \cdots & x_{c_{3}, 18}=0 & x_{c_{3}, 19}=1
\end{array}\right)
$$

whereas the $\mathbf{u}$ matrix can be written as:

$$
\mathbf{u}_{19 \times 11}=\left(\begin{array}{ccc}
P_{g_{2}, 1} & \cdots & P_{g_{2}, 19} \\
P_{g_{3}, 1} & \cdots & P_{g_{3}, 19} \\
V_{g_{1}, 1} & \cdots & V_{g_{1}, 19} \\
V_{g_{2}, 1} & \cdots & V_{g_{2}, 19} \\
V_{g_{3}, 1} & \cdots & V_{g_{3}, 19} \\
P_{c_{2}, 1} & \cdots & P_{g_{2}, 19} \\
P_{c_{3}, 1} & \cdots & P_{g_{3}, 19} \\
V_{c_{1}, 1} & \cdots & V_{c_{1}, 19} \\
V_{c_{2}, 1} & \cdots & V_{c_{2}, 19} \\
V_{c_{3}, 1} & \cdots & V_{c_{3}, 19} \\
U_{c_{1}, 1} & \cdots & U_{c 1,19}
\end{array}\right)^{T}
$$

In case of a regular OPF, just the last column of (8) and the first column of (9) are needed, whereas in case of a preventive SCOPF the whole $\mathbf{x}$ matrix and the first column of (9) are needed. The same reasoning can be applied to the larger power system to obtain its $\mathbf{x}$ and $\mathbf{u}$ matrices.

Table I shows the optimization variables and contingencies scenarios for the different study cases. 
TABLE I

CONTINGENCY SCENARIOS AND CONTROL VARIABLES IN THE DIFFERENT CASE STUDIES.

\begin{tabular}{lrr}
\hline \hline Network & 9AC+3DC & 30AC+5DC \\
\hline N-1 scenarios & 18 & 58 \\
Control variables OPF or preventive SCOPF & 11 & 21 \\
Control variables corrective SCOPF & 209 & 1239 \\
\hline \hline
\end{tabular}

\section{Results}

To solve the power flows for the ac and the dc networks in the presented methodology, the MATPOWER package was used. Instead, to solve the optimization problem, the NOMAD algorithm (Nonlinear Optimization using the MADS Algorithm) was employed. NOMAD is released under the GNU lesser general public license (LGPL) and is available as a solver in the OPTI Toolbox suite for MATLAB, which is a collection of compiled C-MEX functions to solve optimization problems.

Table II displays the cost of operation for the different security operation modes proposed: normal OPF, preventive SCOPF and corrective SCOPF. It also provides a comparison in case the dc network was not in operation.

TABLE II

COST OF OPERATION FOR THE DIFFERENT SECURITY OPERATION MODES.

\begin{tabular}{lccc}
\hline \hline \multicolumn{4}{c}{$9 \mathrm{AC}+3 \mathrm{DC}$ Network } \\
\hline Operation Mode & $\begin{array}{c}\text { Operation } \\
\text { Cost [\$/hr] }\end{array}$ & $\begin{array}{c}\text { Operation } \\
\text { Cost [\%] }\end{array}$ & $\begin{array}{c}\text { Total } \\
\text { Violations }\end{array}$ \\
\hline Normal OPF & $5,244.37$ & $100.00 \%$ & 23 \\
Preventive SCOPF & $5,266.32$ & $100.42 \%$ & 0 \\
Corrective SCOPF & $5,246.65$ & $100.04 \%$ & 0 \\
Without DC Network & $5,296.69$ & $101.00 \%$ & - \\
\hline & $30 \mathrm{AC}+5 \mathrm{DC}$ Network & 10 \\
\hline Normal OPF & 573.01 & $100.00 \%$ & 4 \\
Preventive SCOPF & 573.91 & $100.16 \%$ & 3 \\
Corrective SCOPF & 573.67 & $100.12 \%$ & - \\
Without DC Network & 576.89 & $100.68 \%$ & \\
\hline \hline
\end{tabular}

\section{A. $9 A C+3 D C$ Network}

For the smaller network $(9 \mathrm{AC}+3 \mathrm{DC})$, the cost of operation is $5,296.69 \$ / \mathrm{hr}$ in case the dc network is not in operation and $5,244.37 \mathrm{\$} / \mathrm{hr}$ in case the dc network is in operation and the combined power system is operated in the normal OPF mode. The difference between these two scenarios amounts to $1 \%$ of the total operational costs, which would represent yearly circa $460 \mathrm{k} \$$ in savings if the different stayed constant throughout the year.

In case a preventive or corrective SCOPF are performed, the cost of security is calculated as the difference between the operation cost with and without the added $\mathrm{N}-1$ security. When the network is operated with a normal OPF, there can be 23 different violations to operational constraints in case of contingencies. Instead, when the $9 \mathrm{AC}+3 \mathrm{DC}$ network is operated with a preventive or corrective SCOPF no violations to operational constraints have been found for all the possible 18 $\mathrm{N}-1$ contingencies. However, the network operation becomes slightly more expensive: the extra cost of security is $0.42 \%$ for the preventive SCOPF operation and $0.04 \%$ for the corrective SCOPF operation.

Figure 5 shows the bus voltages, the generator loadings and the line loadings for the normal OPF, preventive SCOPF and corrective SCOPF operation for the 9AC+3DC network. The results are shown as $\mathrm{N}+1$ bars, one for each of the $\mathrm{N}$ possible contingency scenarios and the last one for when the network is in its normal operation state.

If the network would be operated with a normal OPF, in some of the $\mathrm{N}-1$ contingency scenarios there would be maximum voltage violations in buses $1,3,4,6$ and 8 (see Figure 5 (a)). Regarding the line loadings, lines 10 and 11 (which are the first and second dc lines) would get very close to their operational limits, however without violation (99.9\% loading), when there would be a fault on line 11 and 10, respectively (see Figure 5 (d)). Concerning the ac generators and ac-dc power converters, the loading of VSC1 ("generator" 4) would become violated in most of the N-1 contingency scenarios. This is probably due to the fact that VSC1 is the converter controlling the direct voltage in the $\mathrm{dc}$ network, therefore, after a fault, if no other control action is taken, this converter has to absorb any power imbalances to make sure the dc network remains stable.

If the AC9+3DC network would be operated in a preventive SCOPF mode, Figures 5 (b), (e) and (h) show that there would be no violation of operational network limits in case of single contingencies. However, as aforementioned, the cost of operation would be the highest amongst the three analyzed possibilities and, Figure 5 (b) shows that the preventive SCOPF operation mode leads to a lower bus voltage profile in this network, which translate into higher losses. In fact, the total ac and dc losses for the preventive SCOPF operation are $1.916 \mathrm{MW}$, in comparison with $1.173 \mathrm{MW}$ for the normal OPF and 1.270 MW for the preventive SCOPF operation.

Figures 5 (c), (f) and (i) display the network bus voltages, the line loadings and the generators and power converter loadings for the corrective SCOPF operation. As it was the case with the preventive SCOPF operation, there are no operational limit violations for all possible $\mathrm{N}-1$ contingency scenarios. Additionally, the corrective SCOPF mode has a lower operational cost than the preventive SCOPF mode. However, to accomplish this lower operational cost while maintaining the network security, this mode of operation leads to an increased complexity as different operational set points must be sent to the power converters for each of the possible $\mathrm{N}-1$ contingency scenarios. Therefore, it is important to evaluate whether the increase control complexity does not significantly reduce, or offset, the possible gains obtained with the optimization of the unit commitment.

\section{B. $30 A C+5 D C$ Network}

The ac-dc hybrid network in this study case is based on the IEEE 30-bus test system, which is modelled after the electric power system in the Midwest of the USA in 1961. 


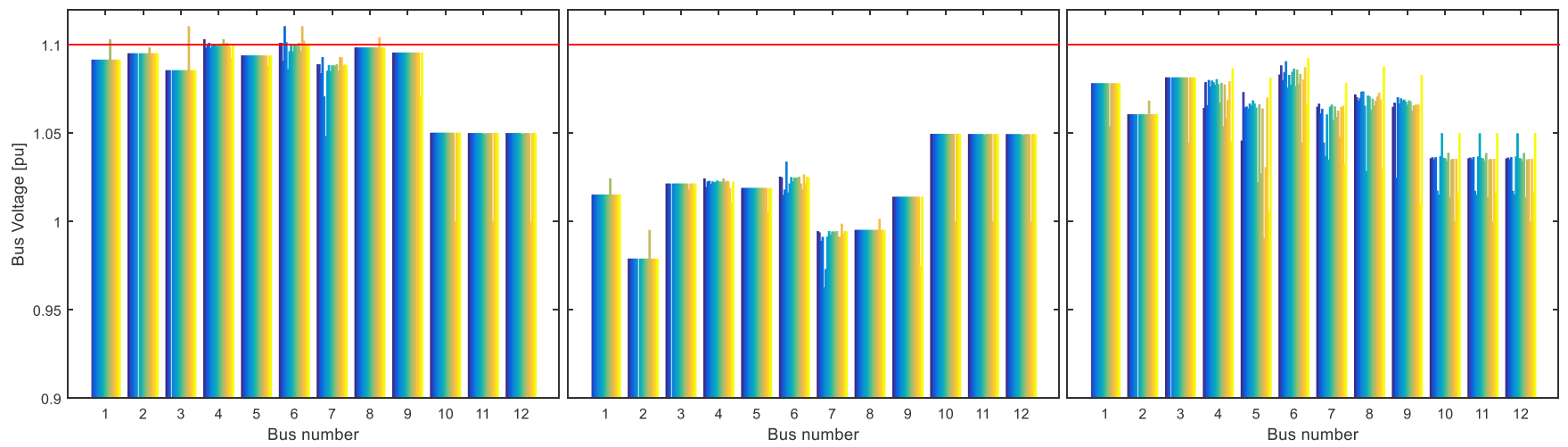

(a) Bus voltages under normal OPF

(b) Bus voltages under preventive SCOPF

(c) Bus voltages under corrective SCOPF
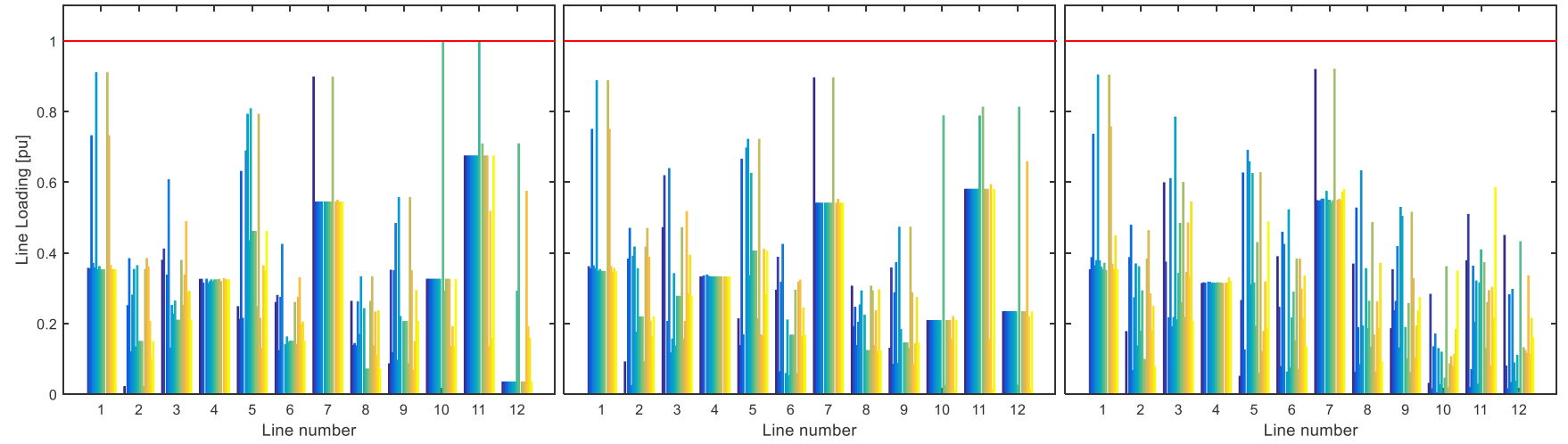

(d) Line loadings under nomal OPF

(e) Line loadings under preventive SCOPF

(f) Line loadings under corrective SCOPF

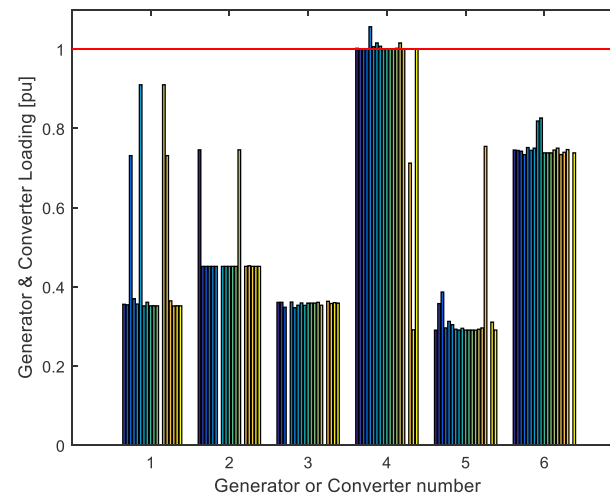

(g) Gen\&Conv loadings under normal OPF

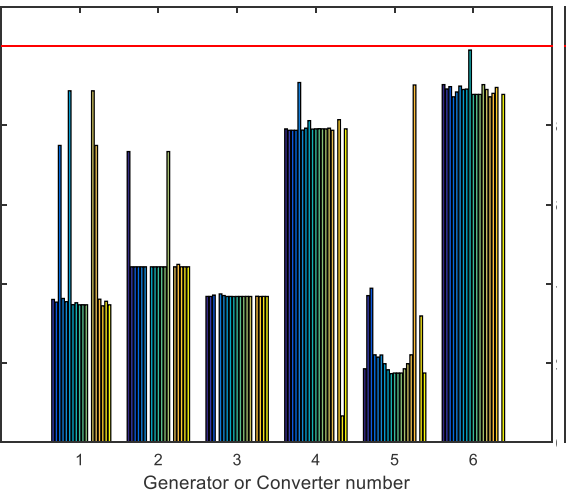

(h) Gen\&Conv loadings under preventive SCOPF

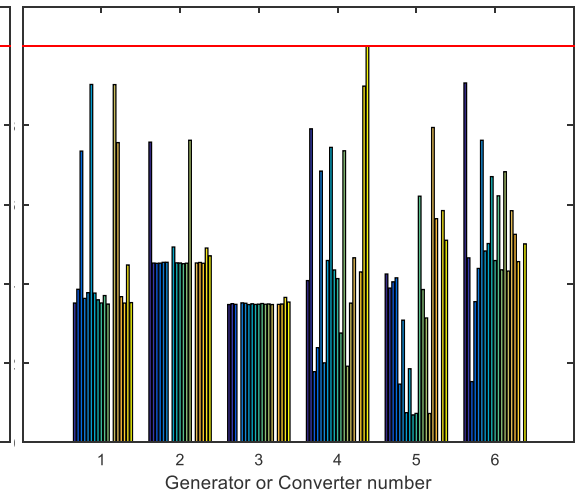

(i) Gen\&Conv loadings under corrective SCOPF

Fig. 5. Bus voltages, the generator loadings and the line loadings for the normal OPF, preventive SCOPF and corrective SCOPF operation for the $9 \mathrm{AC}+3 \mathrm{DC}$ network.

Although the $30 \mathrm{AC}+5 \mathrm{DC}$ network has double the number of generators than the smaller $9 \mathrm{AC}+3 \mathrm{DC}$ network, the operational costs are much lower in the case of the former (see Table II). This is because the ac generators second order polynomial model coefficients are lower in the larger network (circa 5 times lower for the quadratic coefficient) and this network has also less load: whereas the 9AC+3DC network has a load of $315 \mathrm{MW}$, the load in the $30 \mathrm{AC}+5 \mathrm{DC}$ network is only $189.2 \mathrm{MW}$.

Indeed, in comparison with the normal OPF operation for this network, the cost of security in case of preventive or corrective SCOPF operation is of only $0.16 \%$ and $0.12 \%$, respectively; whereas the operational cost without the dc network would be $0.68 \%$ higher. This shows that in both power systems the embedded dc network brings an advantage from the operational costs perspective but also from an operational security point of view.

Figure 6 shows the bus voltages, the generator loadings and the line loadings for the normal OPF, preventive SCOPF and corrective SCOPF operation for the 30AC+5DC network. For the normal OPF operation, there were a total of only 10 possible network violations -4 maximum voltage, 4 maximum line loading and 2 maximum generator loading violations - for the 58 possible $\mathrm{N}-1$ contingency scenarios, which is probably due to the fact that this network is not heavily loaded (the total generation capacity is equal to $335 \mathrm{MW}$, but the total load is 189.2 MW).

Figure 6 (a) shows that the four voltage limit violations 


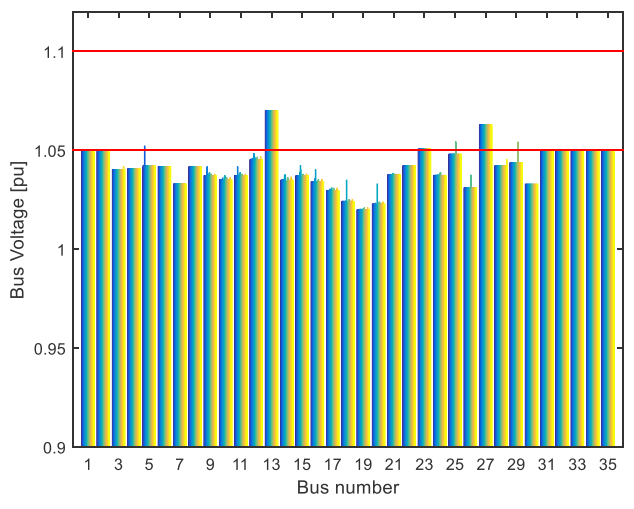

(a) Bus voltages under normal OPF

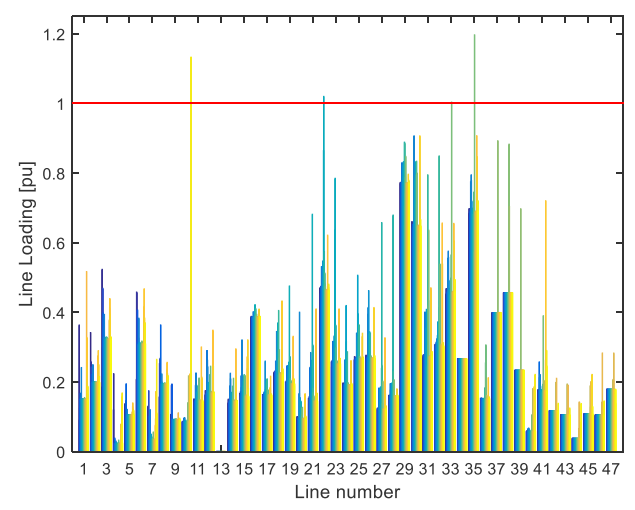

(d) Line loadings under nomal OPF

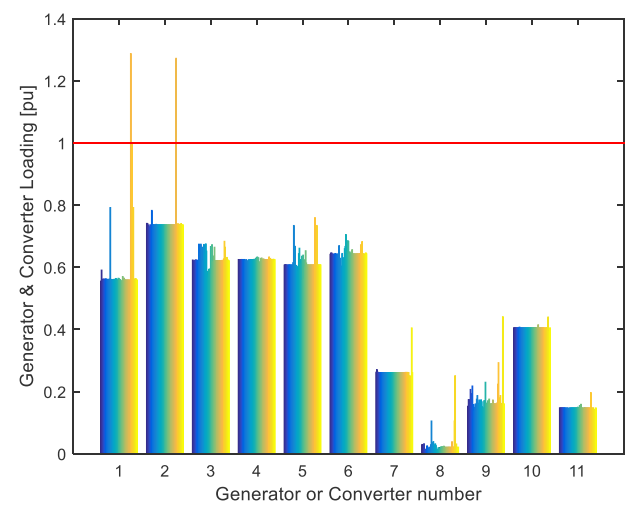

(g) Gen\&Conv loadings under normal OPF

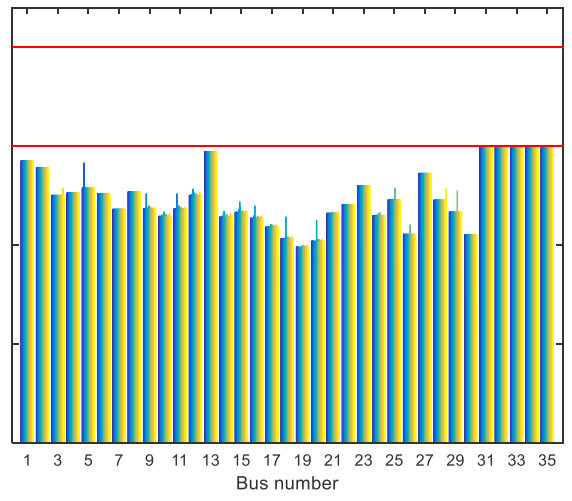

(b) Bus voltages under preventive SCOPF

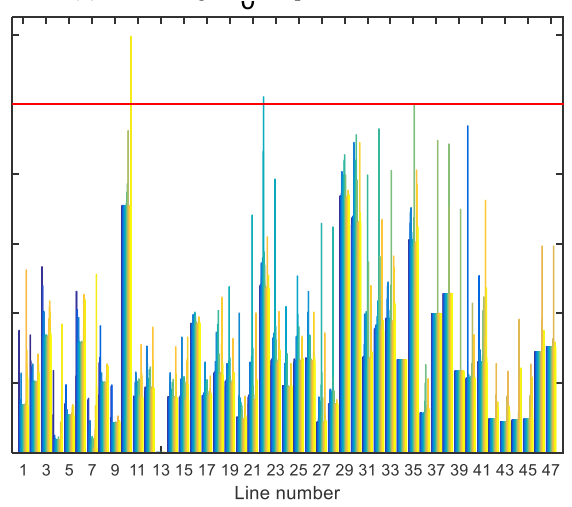

(e) Line loadings under preventive SCOPF

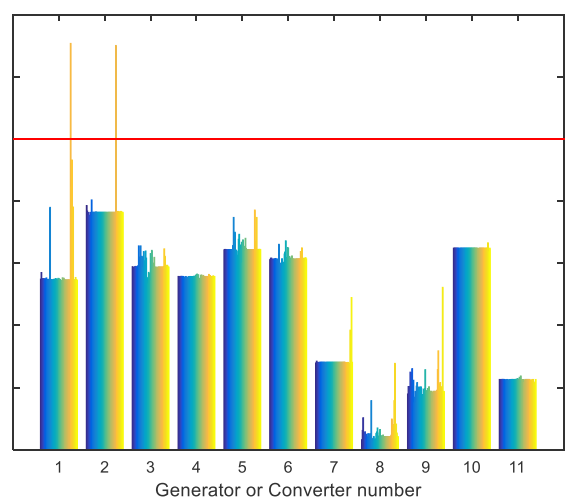

(h) Gen\&Conv loadings under preventive SCOPF

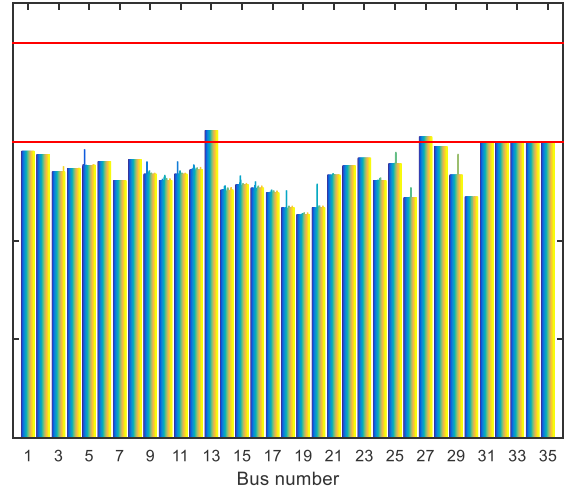

(c) Bus voltages under corrective SCOPF

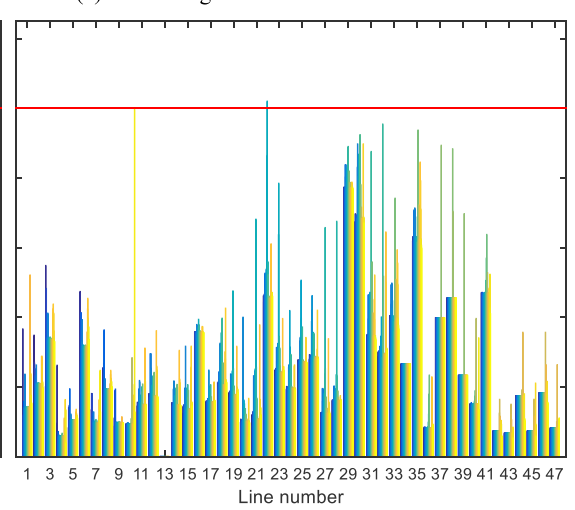

(f) Line loadings under corrective SCOPF

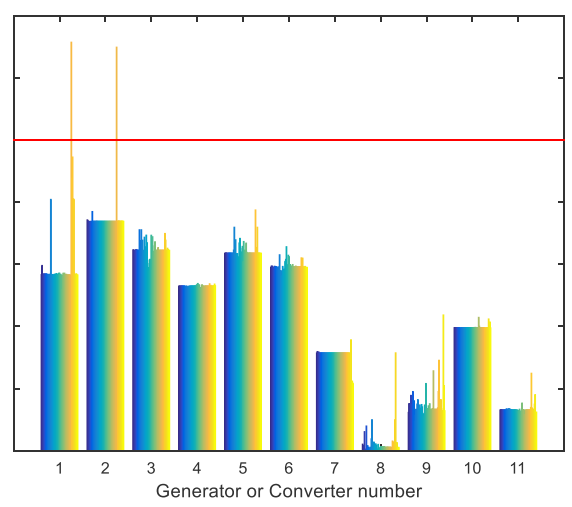

(i) Gen\&Conv loadings under corrective SCOPF

Fig. 6. Bus voltages, the generator loadings and the line loadings for the normal OPF, preventive SCOPF and corrective SCOPF operation for the $30 \mathrm{AC}+5 \mathrm{DC}$ network.

happened in buses 5, 25 (twice) and 29 for faults in ac lines 8 (bus 5), ac lines 33 and 34 (bus 25), and ac line 39 (bus 29). All buses have a limit of $1.05 \mathrm{pu}$, with the exception of the ac generators buses $(1,2,3,13,23$ and 27) where the limit is $1.10 \mathrm{pu}$, Therefore, Figure 6 (b) and (c) show that both the preventive SCOPF and the corrective SCOPF operation successfully managed to reduce the voltage limit violations to zero in case of $\mathrm{N}-1$ contingencies.

Regarding the four line loading violations for the normal OPF operation, these happened on ac lines 22 , when there is a fault on ac line 25; ac lines 33 and 35 , when there is a fault on line 36; and ac line 10 when there is a fault on the ac-dc converter 4 . The preventive SCOPF algorithm managed to eliminate the line loading violations on ac lines
33 and 35, whereas the corrective SCOPF algorithm - since it can alter the power electronic converter set points - also managed to eliminate the violation on ac line 10 when the acdc converter 4 has a fault. However, both the preventive and the corrective SCOPF algorithms did not manage to eliminate the line loading violation on ac line 22 due to a fault on ac line 25 , tough that violation is not of concern since it is of only $2.1 \%$. It is worth noting that ac line 22 is amongst the weakest lines in this network with a power rating of only $16 \mathrm{MW}$ and, therefore, a $2.1 \%$ violation represents only $0.336 \mathrm{MW}$.

Finally, Figure $6(\mathrm{~g})$, (h) and (i) show that there are two violation to the loadings of generators 1 and 2 which could not be meliorated either by the preventive or the corrective SCOPF. The loading limit violation of generator 1 happens 
exactly when the generator 2 has a fault and, viceversa, the one on generator 2 happens instead when the generator 1 has a fault. This is due to the fact that both generators are connected to each other via a strong ac line (ac line 1 has a maximum rating of $130 \mathrm{MW}$ ) and that both generators are functioning as slack nodes of the ac network when the other generator has a fault. Therefore, as mentioned in (1), as these generators are working as slack nodes when the other has a fault, the optimization preventive SCOPF and the corrective SCOPF algorithms cannot control their active power in that specific contingency scenario.

\section{CONCLUSION}

This paper proposed a new methodology for studying the security-constrained optimal power flow operation of $\mathrm{dc}$ networks to support ac networks under contingencies. The obtained results have shown that the power system outage management system works well both in case of the preventive or corrective SCOPF algorithms. For both the analyzed power systems, one with 12 buses $(9 \mathrm{AC}+3 \mathrm{DC})$ and another with 35 buses $(30 \mathrm{AC}+5 \mathrm{DC})$, the corrective SCOPF operation was the one which provided the most security with the lower operational costs. In the first network, where the total network violations were reduced from 23 to none, the extra security costs due to the corrective SCOPF operation was only $0.04 \%$ versus $0.12 \%$ for the second network, where the violations were reduced from 10 to 3 . However, it is important to assess if the higher control complexity in case of the corrective SCOPF operation does overcomes the possible gains obtained with the reduction of the power system operational costs. In that case, a preventive SCOPF operation is the better solution because it improves the security of supply and the power system resilience to outages albeit with higher operational costs than the corrective SCOPF operation. Future work will concentrate on using the binary outage matrix, which is the strongest point of the proposed methodology, combined with a mixed-integer non-linear optimization algorithm to study how the dc network can provide support to the ac network in case of a partial or complete power system restoration.

\section{REFERENCES}

[1] G. F. Reed, "DC Technologies: Solutions to Electric Power System Advancements," IEEE Power and Energy Magazine, pp. 10-17, Nov./Dec. 2012, Guest Editorial.

[2] D. Boroyevich, I. Cvetkovic, D. Dong, R. Burgos, F. Wang, and F. Lee, "Future electronic power distribution systems a contemplative view," in 12th International Conference on Optimization of Electrical and Electronic Equipment. IEEE, May 2010, pp. 1369-1380.

[3] S. Taggart, G. James, Z. Dong, and C. Russell, "The Future of Renewables Linked by a Transnational Asian Grid," Proceedings of the IEEE, vol. 100, no. 2, pp. 348-359, 2012.

[4] M. Aredes, A. F. Da Cunha De Aquino, C. Portela, and E. Watanabe, "Going the distance - Power-Electronics-Based Solutions for LongRange Bulk Power Transmission," IEEE Industrical Electronics Magazine, no. March, p. 13, Mar. 2011

[5] J. Rimez and R. Belmans, "A combined AC/DC optimal power flow algorithm for meshed AC and DC networks linked by VSC converters," International Transactions on Electrical Energy Systems, 2014.

[6] R. Wiget and G. Andersson, "Optimal power flow for combined ac and multi-terminal hvdc grids based on vsc converters," in Power and Energy Society General Meeting, 2012 IEEE. IEEE, 2012, pp. 1-8.

[7] M. Bucher, R. Wiget, G. Andersson, and C. Franck, "Multiterminal hvdc networks: What is the preferred topology?" Power Delivery, IEEE Transactions on, vol. 29, no. 1, pp. 406-413, Feb 2014
[8] M. Aragüés-Peñalba, A. E. Alvarez, S. Galceran-Arellano, and O. Gomis-Bellmunt, "Optimal power flow tool for mixed high-voltage alternating current and high-voltage direct current systems for grid integration of large wind power plants," IET Renewable Power Generation, August 2015

[9] J. Beerten, S. Cole, and R. Belmans, "Generalized steady-state vsc mtdc model for sequential ac/dc power flow algorithms," Power Systems, IEEE Transactions on, vol. 27, no. 2, pp. 821-829, May 2012.

[10] N. Perrier, B. Agard, P. Baptiste, J.-M. Frayret, A. Langevin, R. Pellerin, D. Riopel, and M. Trépanier, "A survey of models and algorithms for emergency response logistics in electric distribution systems. part ii: Contingency planning level," Computers \& Operations Research, vol. 40, no. 7, pp. $1907-1922,2013$.

[11] V. Akhmatov, M. Callavik, C. Franck, S. Rye, T. Ahndorf, M. Bucher, H. Muller, F. Schettler, and R. Wiget, "Technical guidelines and prestandardization work for first hvdc grids," Power Delivery, IEEE Transactions on, vol. 29, no. 1, pp. 327-335, Feb 2014.

[12] T. Haileselassie and K. Uhlen, "Power system security in a meshed north sea hvdc grid," Proceedings of the IEEE, vol. 101, no. 4, pp. 978-990, April 2013.

[13] A. Lotfjou, M. Shahidehpour, Y. Fu, and Z. Li, "Security-constrained unit commitment with ac/dc transmission systems," Power Systems, IEEE Transactions on, vol. 25, no. 1, pp. 531-542, Feb 2010.

[14] S. Barsali, R. Salvati, and R. Zaottini, "Use of HVDC links for power system restoration," Electric Power Systems Research, vol. 79, no. 6, pp. $973-983,2009$.

[15] T. Nagata and H. Sasaki, "A multi-agent approach to power system restoration," Power Systems, IEEE Transactions on, vol. 17, no. 2, pp. 457-462, 2002.

[16] S. Curčić, C. Özveren, L. Crowe, and P. Lo, "Electric power distribution network restoration: a survey of papers and a review of the restoration problem," Electric Power Systems Research, vol. 35, no. 2, pp. 73-86, 1995.

[17] N. Perrier, B. Agard, P. Baptiste, J.-M. Frayret, A. Langevin, R. Pellerin, D. Riopel, and M. Trépanier, "A survey of models and algorithms for emergency response logistics in electric distribution systems. part i: Reliability planning with fault considerations," Computers \& Operations Research, vol. 40, no. 7, pp. 1895 - 1906, 2013.

[18] F. Capitanescu, "Enhanced risk-based scopf formulation balancing operation cost and expected voluntary load shedding," Electric Power Systems Research, vol. 128, pp. 151-155, 2015.

[19] J. Cao, W. Du, and H. Wang, "An improved corrective security constrained opf for meshed ac/dc grids with multi-terminal vsc-hvdc," Power Systems, IEEE Transactions on, vol. PP, no. 99, pp. 1-11, 2015.

[20] R. Yao, X. Zhang, S. Huang, S. Mei, Z. Zhang, X. Li, and Q. Zhu, "Cascading outage preventive control for large-scale ac-dc interconnected power grid," in PES General Meeting - Conference Exposition, 2014 IEEE, July 2014, pp. $1-5$.

[21] F. Capitanescu, J. M. Ramos, P. Panciatici, D. Kirschen, A. M. Marcolini, L. Platbrood, and L. Wehenkel, "State-of-the-art, challenges, and future trends in security constrained optimal power flow," Electric Power Systems Research, vol. 81, no. 8, pp. 1731 - 1741, 2011.

[22] A. Attarha and N. Amjady, "Solution of security constrained optimal power flow for large-scale power systems by convex transformation techniques and taylor series," IET Generation, Transmission \& Distribution, 2016.

[23] Y. Liu and M. Ferris, "Security-constrained economic dispatch using semidefinite programming," in 2015 IEEE Power Energy Society General Meeting, July 2015, pp. 1-5.

[24] R. Teixeira Pinto, P. Bauer, S. Rodrigues, E. Wiggelinkhuizen, J. Pierik, and B. Ferreira, "A Novel Distributed Direct-Voltage Control Strategy for Grid Integration of Offshore Wind Energy Systems Through MTDC Network," IEEE Transactions on Industrial Electronics, vol. 60, no. 6, pp. $2429-2441$, june 2013.

[25] R. Teixeira Pinto, S. F. Rodrigues, E. Wiggelinkhuizen, R. Scherrer, P. Bauer, and J. Pierik, "Operation and Power Flow Control of Multi-Terminal DC Networks for Grid Integration of Offshore Wind Farms Using Genetic Algorithms," Energies, vol. 6, no. 1, pp. 1-26, 2012. [Online]. Available: http://www.mdpi.com/1996-1073/6/1/1

[26] R. Teixeira Pinto, "Multi-Terminal DC Networks: System Integration, Dynamics and Control," PhD. Thesis, Delft University of Technology, Delft, March 2014, ISBN: 978-94-6203-550-8.

[27] R. D. Zimmerman, C. E. Murillo-Sánchez, and R. J. Thomasi, "Matpower: Steadystate operations, planning and analysis tools for power systems research and education," Power Systems, IEEE Transactions on, vol. 26, no. 1, pp. 12-19, 2011. 